\title{
Estimación del Error en Elementos Hexaédricos
}

\author{
Mary Vergara ${ }^{1}$, Sebastián Provenzano ${ }^{1}$, Carlos Bloem $^{1}$ y Francisco Fuenmayor ${ }^{2}$ \\ (1) Universidad de Los Andes, Facultad de Ingeniería, Escuela de Ingeniería Mecánica, Grupo de \\ Diseño y Modelado de Máquinas (DIMMA), La Hechicera, Mérida-Venezuela \\ (e-mail:vmary@ula.ve, prse@ula.ve,cbloem@ula.ve,) \\ (2) Universidad Politécnica de Valencia, Departamento de Ingeniería Mecánica y Materiales, \\ Camino de Vera s/n, Valencia-España (e-mail: ffuenmay@mcm.upv.es)
}

\section{Resumen}

En este trabajo se utiliza el estimador de error de discretización $Z^{2}$, propuesto por Zienckiewz y Zhu, para aplicar el refinamiento por subdivisión de elementos hexaédricos. La obtención del campo de tensiones mejoradas se basa en técnicas de alisado de tensiones por zonas, con las cuales se desarrollan tres procedimientos que evalúan el error. Los procedimientos desarrollados se aplican a dos problemas con solución conocida, con el objetivo de evaluar y definir las características de cada técnica propuesta. Se observó que en el problema con solución suave se puede utilizar la técnica donde se considera la presencia de nodos ficticios e integración reducida. Sin embargo, en el problema con singularidades es mejor considerar dichos nodos, con una integración completa para la estimación de error.

Palabras clave: estimador de error, error de discretización, alisado de tensiones, adaptatividad-h, índice de efectividad

\section{Error Estimation in Hexahedral Element}

\begin{abstract}
This paper uses the discretization estimator error $Z^{2}$, proposed Zienckiewz and Zhu, for implementing the refinement by subdivision in hexahedrons elements. Obtaining improved tension field is based on techniques of smoothing tensions by zones, developing three procedures for evaluating the error. The procedures developed in this work were applied to two problems with known solution, in order to evaluate and define the characteristics of each proposed technique. In was observed that in the problem with smooth solution the technique that considers the presence of fictitious nodes and reduced integration could be used. However, in the problem with singularities it is better to consider the nodes with full integration for error estimation.
\end{abstract}

Keywords: error estimator, discretization error, smoothed stress, $h$-adaptive, effectivity index 


\section{INTRODUCCIÓN}

Los estimadores de error a posteriori para elementos finitos (EF) utilizan la información obtenida durante el procedimiento análisis, además de suposiciones a priori sobre la solución y permiten realizar estimaciones del verdadero error de discretización (Ainsworth y Oden, 2000). Éstos son los más utilizados en aplicaciones prácticas pues proporcionan una medida cuantitativa del error de discretización, se obtienen tanto a nivel puntual como local y global, se pueden aplicar a ecuaciones diferenciales parabólicas no lineales como en De Frutos et al. (2007) y para su evaluación, es mejor utilizar procedimientos basados en "recovery" (Zienkiewicz, 2006). En este estudio se implementan tres procedimientos para evaluar el error estimado, considerando la presencia de nodos no conformes. El primer procedimiento no considera en la determinación del error la presencia de estos nodos, sin embargo los dos siguientes sí, en éstos, se realiza una integración estándar en dos puntos de gauss (ptg) y una integración reducida para uno sólo. Para encontrar los campos alisados de tensiones necesarios para estimar el error $Z^{2}$, propuesto por Zienkiewicz y Zhu (1987) y debido a que éste necesita de un campo de tensiones mejorado (recovery), se utilizan dos técnicas con una excelente robustez (Boroomand y Mossaiby, 2005), "Superconvergent Patch Recovery" mejorada (SPR-R) y "Recovery by Equilibrium Patches" mejorada (REP-R), las cuales consideran condiciones de contorno y se encuentran implementadas y comparadas entre si en Vergara et al. (2008) y con redes neuronales en Ivirma et al., (2009). Finalmente, con el objetivo de evaluar y definir las características de cada técnica propuesta para la estimación del error se aplican dichos procedimientos a dos problemas con solución conocida, evaluando el índice de efectividad y la convergencia de la solución. Las evaluaciones muestran que se puede utilizar la técnica donde se considera la presencia de nodos ficticios e integración reducida en el problema con solución suave y el mismo tipo de nodos con integración completa si se trata del problema con singularidades.

\section{ERRORES EN LA SOLUCIÓN DE EF Y CONVERGENCIA ASINTÓTICA}

Los estimadores de error a posteriori, se basan en la idea de conseguir una mejor aproximación a la solución exacta para tensiones a partir de la solución de EF y calcular su diferencia como una estimación del error exacto. El estimador $Z^{2}$ asegura su utilidad en análisis prácticos, porque posee las propiedades deseables descritas por Babuska et al. (2000) y consiste en utilizar en la expresión del error en norma energética, en lugar del campo de tensiones exacto, que es desconocido, un campo de tensiones mejorado obtenido a partir del campo de tensiones de EF, como se muestra en la expresión (1):

$\left\|\mathrm{e}_{\mathrm{ex}}\right\| \approx\left\|\mathrm{e}_{\mathrm{es}}\right\|=\left(\int_{\mathrm{V}}\left(\sigma^{*}-\sigma_{\mathrm{ef}}\right)^{\top} \mathbf{D}^{-1}\left(\sigma^{*}-\sigma_{\mathrm{ef}}\right) \mathrm{dV}\right)^{\frac{1}{2}}$

$\sigma^{*}$ : es el campo de tensiones mejorado, $\left\|e_{e s}\right\|:$ el error estimado, $\mathbf{D}:$ la matriz de propiedades del material, $\sigma_{\text {ef }}$ : es el campo de tensiones de elementos finitos según la expresión (2), que contiene el campo de desplazamientos obtenidos de EF $\mathrm{u}_{\mathrm{ef}} \mathrm{y}$ la matriz de deformación-desplazamientos $\mathbf{B}$ :

$$
\sigma_{\text {ef }}=\mathbf{D B u}_{\text {ef }}
$$

La aproximación (1) converge hacia un valor nulo a medida que se va refinando la malla ya que ambos campos de tensiones se acercan cada vez más a la solución exacta del problema. Por ello el estimador tenderá también a producir valores más correctos durante dicho proceso. El error estimado relativo $\eta$ en la norma energética a nivel global se obtiene según:

$\eta=\frac{\left\|e_{\text {es }}\right\|}{\left(\left\|u_{\text {ef }}\right\|^{2}+\left\|e_{\text {es }}\right\|^{2}\right)^{1 / 2}} 100$

Desde un punto de vista práctico, también interesa medir el índice de efectividad " $\theta$ ", el cual representa la relación entre los valores del error estimado $\left\|e_{\mathrm{es}}\right\|$ y el error exacto $\left\|\mathrm{e}_{\mathrm{ex}}\right\|$ y se expresa como: 
$\theta=\frac{\left\|\mathrm{e}_{\mathrm{es}}\right\|}{\left\|\mathrm{e}_{\mathrm{ex}}\right\|}$, con $\left\|\mathrm{e}_{\mathrm{ex}}\right\| \approx \sqrt{\left\|\mathrm{u}_{\mathrm{ex}}\right\|^{2}-\left\|\mathrm{u}_{\mathrm{ef}}\right\|^{2}}$

donde: $\left\|\mathrm{u}_{\mathrm{ex}}\right\|$ es la norma energética de la solución exacta y $\left\|\mathrm{u}_{\mathrm{ef}}\right\|$ la de elementos finitos.

\section{CONVERGENCIA ASINTÓTICA EN LA NORMA ENERGÉTICA}

La expresión que relaciona el error exacto de la solución de elementos finitos y el tamaño de elemento considerando la versión $\mathrm{h}$ del método de elementos finitos esta dada por Craig et al. (1989) como:

$\left\|\mathrm{e}_{\mathrm{ex}}\right\| \approx \mathrm{Ch}^{\min (p, \lambda)}$

donde $\lambda$,es una constante que caracteriza la intensidad de las singularidades y $c$ es un valor positivo que depende del problema estudiado y del grado del polinomio usado en la interpolación, pero no del tamaño de los elementos. Si la solución es suave, el valor de c es grande y entonces la velocidad de convergencia está limitada por el orden polinómico de los elementos, de tal manera que para problemas tridimensionales, la velocidad de convergencia $v$ será:

$v=\frac{1}{3} p$

Para los casos tridimensionales, si se realiza un refinamiento uniforme según lo expuesto para elementos lineales, la velocidad de convergencia tiende a $-1 / 3$ cuando la solución exacta del problema es suave, mientras que, para un caso con fisura se tiene que la velocidad de convergencia es $v=-1 / 6$. Gráficamente la velocidad de convergencia se obtiene como la pendiente de la recta que se consigue al representar el $\log \left(\left\|e_{e x}\right\|\right)$ en función de los gdl.

\section{PROCEDIMIENTOS PARA EL CALCULO DE $\left\|e_{\mathrm{es}}\right\|$ EN LA SUBDIVISIÓN DE HEXAEDROS}

Al resolver problemas con refinamiento por subdivisión con EF, aparecen nodos no conformes, lo que origina que sobre elementos no divididos existan nodos que no tienen contrapartida, como se muestra en la Fig. 1a, donde los nodos a, b y c no tienen contrapartida en elementos adyacentes a los divididos y no divididos respectivamente. Por esto, se desarrollan tres procedimientos para calcular el error estimado. El primero no considera los nodos no conformes para calcular el error y en los otros dos se usan elementos ficticios (ver Fig. 1.b) para considerar la presencia de dichos nodos. El objetivo de desarrollar estos procedimientos, es encontrar cual de ellos presenta numéricamente un mejor comportamiento según las ecuaciones (3) y (4); éstos se describen a continuación:

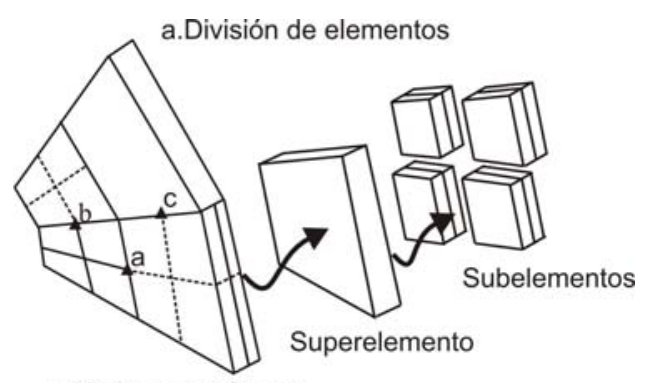

A Nodo no conforme

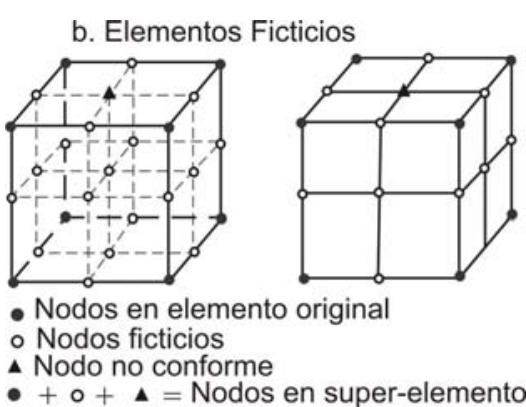

- $+0+\mathbf{\Delta}=$ Nodos en super-elemento

Fig. 1: División de elementos con nodos no conformes y elementos ficticios

Malla con Elementos no Divididos ( $\mathrm{e}_{\mathrm{es}} 0$ SPR-R y REP-R)

Este primer procedimiento, es el más sencillo y consiste en calcular el error estimado utilizando integración estándar para los elementos que posee la malla, sin considerar la presencia de los nodos no 
conformes. La evaluación de la integral de la ecuación (1), se realiza utilizando reglas de integración numéricas en coordenadas locales. A nivel de elemento el error será:

$\left\|e_{e s}\right\|_{e}^{2}=\sum_{i=1}^{N p t g} H_{\xi_{i}} H_{\eta_{i}} H_{\zeta_{i}}\left(\left(\sigma^{*}-\sigma_{e f}\right)^{\top} D^{-1}\left(\sigma^{*}-\sigma_{e f}\right)\right)_{\left(\xi_{i}, \eta_{i}, \zeta_{i}\right)}|J|_{\left(\xi_{i} \eta_{i}, \zeta_{i}\right)}$

donde: $H_{\xi_{i}} H_{\eta_{i}} H_{\zeta_{i}}$ corresponden a los pesos de ptg, $|J|_{\left(\xi_{i}, \eta_{i}, \zeta_{i}\right)}$ el determinante de la matriz jacobiana de la transformación de coordenadas. El número de puntos de integración con sus respectivos pesos son los mismos que los utilizados para obtener la solución de elementos finitos. Por lo tanto Nptg $=2$ para elementos lineales y su magnitud es la unidad. Para estimar el error en toda la malla, se realiza la sumatoria según:

$\left\|\mathrm{e}_{\mathrm{es}}\right\|^{2}=\sum_{\mathrm{i}=1}^{\text {Nelem }}\left\|\mathrm{e}_{\mathrm{es}}\right\|^{2}$

\section{Malla con Elementos Divididos y Nptg $=\operatorname{Nptg}\left(\mathrm{e}_{\mathrm{es} \_} 1\right.$ SPR-RSUBE y REP-RSUBE)}

En esta técnica se considera la presencia de nodos no conformes, dividiendo los elementos adyacentes a los divididos con nodos y elementos ficticios. El error estimado se calcula con la misma cantidad de puntos de integración que la solución obtenida por elementos finitos. Para aplicar esta técnica es necesario: Generar elementos ficticios. Para esto, se prolongan las subdivisiones entre los nodos y elementos que sean necesarios. El elemento que contiene los nodos originales, los ficticios y los no conformes se llamará superelemento y el que se genera debido a la presencia de nodos no conformes será llamado subelemento (ver Fig. 1b). Debido a que se permite un grado de división, para garantizar la partición de toda la malla basta con crear ocho subelementos en cada superelemento con los nodos ficticios que sean necesarios como se muestra en la Fig. 1b. Asignar coordenadas y tensiones en nodos del superelemento. Las tensiones conocidas del elemento original se deben asignar sobre el superelemento en los nodos comunes (nodos vértice del hexaedro y nodos no conformes). Las tensiones asignadas corresponden a las calculadas por EF (extrapoladas hacia los nodos) y a las tensiones mejoradas según la técnica SPR-R y REP-R. Si se definen las tensiones alisadas y las calculadas por elementos finitos en los nodos del superelemento como: $\sigma_{\mathrm{S}}^{*}, \sigma_{\mathrm{S}}$ respectivamente y $\sigma^{*}, \sigma$ son las tensiones en el elemento original entonces:

$\sigma_{\mathrm{s}}^{*}=\sigma^{*}, \sigma_{\mathrm{s}}=\sigma$

Asignar coordenadas y tensiones en nodos ficticios. Una vez que se han definido sobre el superelemento todas las coordenadas y tensiones posibles, se calculan por interpolación, las coordenadas y tensiones en los nodos que faltan, generando así una pequeña red de elementos compuesta por ocho subelementos, cuyas tensiones nodales de EF y mejoradas son conocidas. Calcular tensiones en los puntos de integración numérica. Se realiza una interpolación para calcular las tensiones de $\mathrm{EF}$ “ $\sigma_{\mathrm{se}_{\mathrm{j}}}$ ” y mejoradas " $\sigma_{\mathrm{se}_{\mathrm{j}}}^{*}$ " de cada subelemento en los puntos de Gauss con la finalidad de aplicar la integración numérica en el cálculo del error. Calcular el error estimado en el superelemento. Se utiliza una expresión análoga a la ecuación (7), pero desarrollada para cada subelemento como se observa en la ecuación (10). En este caso el número de puntos de integración corresponden a los utilizados en la solución de EF, así para elementos lineales $\mathrm{Nptg}=2$. Finalmente para obtener el error estimado global, se suman cada uno de los errores estimados en los superelementos en toda la malla, utilizando la ecuación (8).

$\left\|\mathrm{e}_{\mathrm{es}}\right\|_{\mathrm{s}}^{2}=\sum_{\mathrm{j}=1}^{8} \sum_{\mathrm{i}=1}^{\mathrm{Nptg}} \mathrm{H}_{\xi_{\mathrm{i}}} \mathrm{H}_{\eta_{\mathrm{i}}} \mathrm{H}_{\zeta_{\mathrm{i}}}\left(\sigma_{\mathrm{se}_{\mathrm{j}}}^{*}-\sigma_{\mathrm{se}_{\mathrm{j}}}\right) \mathbf{D}^{-1}\left(\sigma_{\mathrm{se}}^{*}-\sigma_{\mathrm{se}}\right)^{\top}|\mathbf{J}|_{\xi_{\mathrm{i}}, \eta_{\mathrm{i}}, \zeta_{\mathrm{i}}}$ 


\section{Malla con Elementos Divididos y Nptg $\neq$ Nptg ( $\mathrm{e}_{\mathrm{es} \_} 2$ SPR-RSUBER y REP-RSUBER)}

Este procedimiento también considera la presencia de nodos no conformes, utilizando para ello una técnica análoga a la presentada anteriormente. La diferencia básica está en que al calcular el error para cada subelemento, se utilizan menos puntos de integración (integración reducida) que los utilizados en la solución de EF; es decir $\mathrm{Nptg}=1$.

\section{RESULTADOS Y DISCUSIÓN}

Los análisis se han desarrollado para problemas en 3D, con elementos hexaédricos lineales, donde el campo de tensiones mejorado $\sigma^{*}$ se obtiene mediante las técnicas SPR-R y REP-R y la $h-$ adaptatividad es el resultado de aplicar el refinamiento por subdivisión de hexaedros en: a. Esfera de pared gruesa sometida a presión interna con: radio interno, $R_{1}=5.10^{-2}(\mathrm{~m})$, radio externo $R_{2}=20.10^{-2}(\mathrm{~m})$, Presión interna $P=1\left(\mathrm{~N} / \mathrm{m}^{2}\right)$, módulo de elasticidad $E=1000\left(\mathrm{~N} / \mathrm{m}^{2}\right), v=0.3$, y energía de deformación exacta $\left\|\mathbf{u}_{\mathrm{ex}}\right\|^{2}=0.1308996939(\mathrm{Nm})^{1 / 2}$. b. Placa cargada a tensión con grieta lateral con: $\sigma=1000\left(\mathrm{~N} / \mathrm{m}^{2}\right)$, longitud de grieta de $a=0.6 .10^{-2}(\mathrm{~m})$, en una placa de espesor $\mathrm{b}=2.10^{-2}(\mathrm{~m})$, ancho $\mathrm{c}=6.10^{-2}(\mathrm{~m})$, alto $\mathrm{d}=1.10^{-2}(\mathrm{~m}), \mathrm{E}=10^{7}\left(\mathrm{~N} / \mathrm{m}^{2}\right), \mathrm{v}=0.333,\left\|\mathrm{u}_{\text {ex }}\right\|^{2}=0.112007(\mathrm{Nm})^{1 / 2}$

Al realizar un análisis de la evolución del índice de efectividad y la convergencia en las Figuras 2 y 3 , se observa que las técnicas SPR-R y REP-R presentan un comportamiento análogo entre si, lo que diferencia la tendencia de $\theta$ y $\eta \%$ es el procedimiento utilizado en la estimación del error: ${ }^{e_{e s}{ }_{-}}{ }^{\text {, }}$ $e_{e s-1} y^{e_{e s}{ }^{2}}$, que varia de forma más notable.
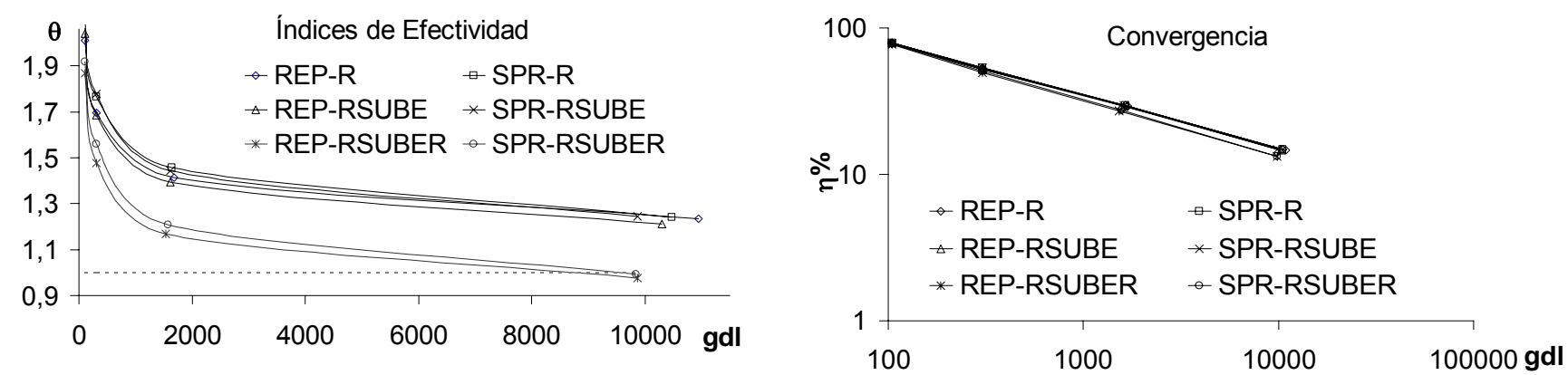

Fig. 2: Índice de Efectividad y Convergencia para la Esfera con Presión Interna
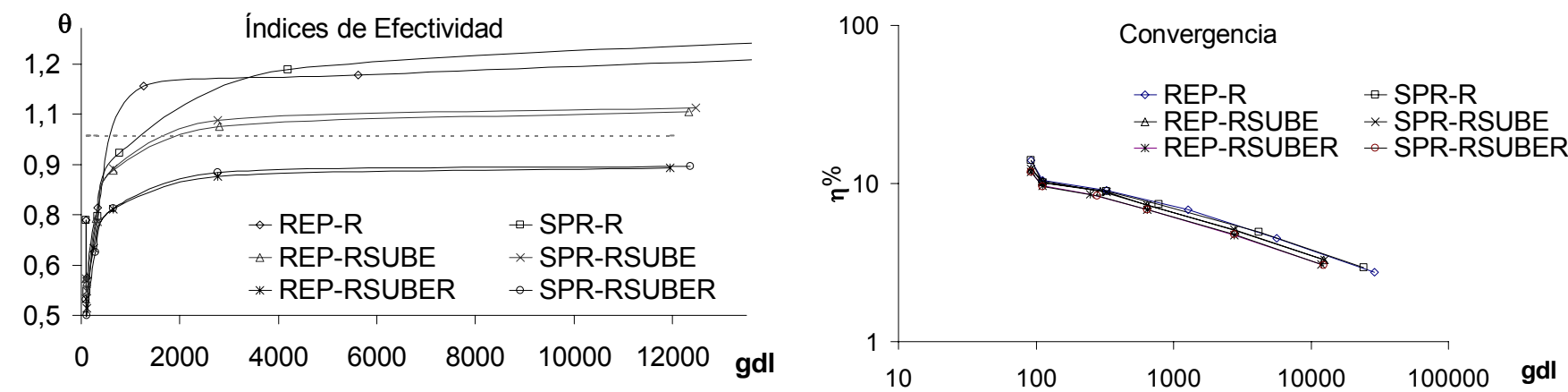

Fig. 3: Índice de Efectividad y Convergencia para la Placa con grieta lateral

En la Figura 2 se observa que cuando se utiliza para la estimación del error la integración reducida con subelementos, el valor de $\theta$ más cercano a la unidad se encuentra a partir de la tercera malla, mientras que para las otras técnicas con integración estándar esta magnitud se encuentra mas aleja- 
do, igual ocurre con la convergencia que es mayor con el ${ }^{e_{e s}{ }^{2}}$ que con las otras técnicas. En la Figura 3, el índice de efectividad con las técnicas: ${ }^{e_{e s}{ }_{-}}{ }, e_{e s}{ }^{2}$, tiende a estar mas alejado de la unidad a partir de la cuarta malla, mientras que con ${ }^{e_{e s}-1}$ el ${ }^{\theta}$ se encuentra más cercano al valor deseado. La velocidad de convergencia es ligeramente mayor para este mismo caso cuando se aumentan los gdl.

\section{CONCLUSIONES}

En general, de los ejemplos analizados para cada una de las técnicas desarrolladas de estimación de error, se puede concluir que: cuando se considera el problema con solución suave, la técnica desarrollada para elementos que consideran la presencia de nodos no conformes " $e_{\mathrm{es}} 2_{2}$ " e integración reducida, presenta valores de fiabilidad que se incrementan progresivamente hasta alcanzar el valor unitario, que es el valor deseado. La velocidad de convergencia en pares de malla en el proceso adaptativo, también presenta información interesante pues en este problema, ésta tiende aproximadamente a $v \approx-1 / 3$, por lo tanto puede establecerse una buena evolución de $v$ en el proceso adaptativo utilizando la técnica $e_{\mathrm{es}} 2$. Cuando se considera el problema con solución singular, es preferible utilizar el procedimiento donde se construyen elementos ficticios " $e_{\mathrm{es}}{ }_{1}$ " e integración estándar, ya que aquí el índice de efectividad unitario se alcanza con menores gdl, manteniéndose en refinamientos sucesivos, con una $v \approx-1 / 3$ para todos los casos estudiados, lo que implica que este tiende a un valor cercano al que presentan los problemas con solución suave.

\section{REFERENCIAS}

Ainsworth, M. y T. Oden; A posteriori error estimation in Finite Element Analysis, John Wiley \& Sons, EEUU, pp xiv-xiii, 397-397 (2000)

Babuska, I., T. Strouboulis, D. Dibyendu, y S. Gangaraj; What do we want and what do we have in a posteriori estimates in the FEM, The Mathematics of Finite Elements and Applications X., Proceedings of the 10th conference, Brunel Univ., Uxbridge, Middlesex, GB, Junio 22-25, 1999. Amsterdam: Elsevier. 163-180 (2000).

Boroomand B. y F. Mossaiby; Generalization of robustness test procedure for error estimators. Part II: test results for error estimators using SPR and REP, Int. J. Numer. Meth. Eng: 64(1), 461-502 (2005).

Craig, A. W., M. Ainsworth, J. Zhu, y O. Zienkiewicz; $h$ and $h-p$ version error estimation and adaptive procedures from theory to practice, Engineering with Computers: 5(1), 221-234 (1989).

De Frutos J., B. Garcia, J. Novo; A posteriori error estimates for fully discrete nonlinear parabolic problems, Comput. Methods Appl. Mech. Engrg: 196(1), 3462-3474 (2007)

Ivirma, L. y otros cinco autores; Artificial Neural Networks application for Stress Smoothing in Hexaedrons, 10th WSEAS International Conference on Nueral Networks, 4 -46, Prague, Czech Republic, March 23-25 (2009)

Vergara. M., S. Provenzano, C. Bloem y R. Chacón; Diferentes Técnicas para el Alisado de Tensiones 3D, Información Tecnológica: 19(5), 111-118 (2008)

Zienkiewicz, O.; A The background of error estimation and adaptivity in finite element computations, Comput. Methods Appl. Mech. Engrg.: 195, 207-213 (2006)

Zienkiewicz, O. y J. Zhu; A simple error estimator and adaptive procedure for practical engineering analysis, International Journal Numerical Methods in Engineering: 24,337-357 (1987) 